\title{
Responsabilidad social de las empresas: ¿Una solución para el desarrollo en América Latina?
}

\author{
Bull, Benedicte* \\ * Investigadora del Centro para el Desarrollo y el Medio Ambiente, Universidad de Oslo \\ P.B. 1116 Oslo, Noruega. E- mail: benedicte.bull@sum.uio.no
}

\section{Resumen}

La responsabilidad social de las empresas (RSE) se ha constituido en un tema de discusión y análisis para distintos actores vinculados a diferentes movimientos de activistas, así como por parte de la comunidad empresarial. En este artículo se reflexiona sobre las varias vertientes de estudio que están relacionadas con el auge que viene teniendo la RSE. Se argumenta que para adelantar un análisis de la RSE como una herramienta de desarrollo hay que incluir una tercera perspectiva: la de la economía política. La hipótesis de la cual se parte es que la conducta de la RSE depende de dos vertientes: a) La que está relacionada con el sector económico y rama de producción a la que pertenecen y b) La que proviene de las relaciones entre el Estado y las empresas. Se concluye que a pesar de las exageradas literaturas existentes sobre la RSE se debe estudiar más y analizar la relación Estado-empresa; aquí sólo se ha propuesto la inclusión de la economía política pero queda pendiente esclarecer analíticamente por qué algunas empresas toman mas responsabilidad social que otras.

Palabras clave: Responsabilidad social de las empresas, economía política, ética, Estado.

\section{Social Responsibility of the Companies: A Solution for the Development in Latin America?}

\begin{abstract}
Social responsibility in business (RSE) has become a theme for discussion and analysis among many actors in activist movements, as well as in the business community. This article comments various areas of study that are related to this upsurge in RSE. It is argued that in order to advance in the study of RSE as a development tool, it is necessary to include a third perspective: that of political economy. The hypothesis on which this is based is that RSE conduct depends on two relationships: a) the relationship of the
\end{abstract}


economic sector and the productive sector to which a business pertains, and b) the relationship between the state and the business sector. The conclusion is that in spite of the exaggerated emphasis on RSE, it is necessary to study more profoundly the relationship government-business. In this paper we have focused on the proposal to include business in the political economy. It is still necessary to analytically study why certain businesses are more socially responsible than others.

Key words: Social responsibility of businesses, political economy, ethics, state.

Recibido: 04-02-06. Aceptado: 04-10-04

\section{Introducción}

La idea de "la responsabilidad social de las empresas" (RSE) es actualmente adoptada por muchos de los actores y sectores del contexto político internacional. Muchos grupos; tanto movimientos activistas, como por grandes participes de la comunidad empresarial, instituciones financieras y agencias de desarrollo, lo ven como una manera de mejorar la calidad de vida de la gente. Al mismo tiempo que se puede alcanzar la protección del medio ambiente.

En el contexto Latinoamericano, la RSE se ha lanzado como una herramienta de competitividad y desarrollo (Vives, 2004; Peinado-Vara, 2004). Las dos perspectivas teóricas dominantes en el debate sobre RSE son, por un lado, la ética empresarial, y por otro, la gestión empresarial. Bajo la primera perspectiva teórica se discuten los argumentos éticos a favor y en contra de la idea acerca de que las empresas tienen una responsabilidad social que va más allá de producir un superávit y de cumplir con las leyes. Bajo la segunda perspectiva teórica, el propósito más importante de asumir una responsabilidad social, no solamente tiene un aspecto ético, sino que también es un buen negocio. Entonces el debate tiene que ver con métodos para incluir la RSE en las operaciones empresariales.

Este artículo tiene como intención argumentar que para analizar la RSE como una herramienta de desarrollo hay que incluir una tercera perspectiva, la de economía política. En el debate actual, frecuentemente se excluye esta perspectiva, porque como señala Phillips (1993), la ética empresarial toma la economía capitalista como punto de partida, mientras que la economía política se pregunta o investiga cuál es el mejor sistema económico. Sin embargo, esto es una visión muy limitada sobre economía política. Una explicación más amplia se encuentra en las diferencias. La ética empresarial cree en el 
proceso de reflexión ético de las empresas como una manera de mejorar el estado de las cosas, mientras que la economía política pretende investigar como nuestras sociedades pueden organizarse para protegerse de las consecuencias más negativas de la búsqueda de ganancia de las empresas $y$, al mismo tiempo beneficiarse de las actividades capitalistas.

Por eso debemos preguntarnos: ¿Dentro de que condiciones sociales y económicas va a ser más probable que las empresas tomen una responsabilidad social? El propósito de este artículo es diseñar un marco analítico de la RSE basado en la economía política, formular algunas hipótesis iniciales, y elaborar una prueba preliminar, basándome en los datos existentes. Las hipótesis formuladas son: Que la conducta de la RSE de las empresas depende (a) del sector económico y ramas de producción, y (b) de las relaciones entre el estado y las empresas. Por lo cual, para que la RSE pueda ser utilizada como una herramienta de desarrollo, tiene que ser discutida conjuntamente en escenarios donde se planteen propuestas sobre modelos de desarrollo y regulaciones generales.

\section{Como definir la RSE}

¿Que significa la responsabilidad social empresarial? Aunque el concepto RSE es relativamente reciente, la idea sobre la responsabilidad social que tienen las empresas no es nueva. Unos autores señalan que las raíces de RSE se encuentran en las leyes de Judea del tiempo pre-Cristiano (Klausen, 2000). Otros han encontrado información sobre la gestión empresarial en los archivos de los Estados Unidos entre los años 1950 y 1960 (Melling y Jensen, 2002). Ahí la responsabilidad social fue considerada como una obligación moral y una responsabilidad personal del dueño de la empresa (Bowen, 1953; Davis, 1963).

Sin embargo, el debate actual no proviene de un contexto nacional, sino del auge de las grandes corporaciones transnacionales, y el paralelo surgimiento de una globalización de los medios de comunicación y Organizaciones No-Gubernamentales (ONGs) con extensiones globales (Holliday et al., 2002; Jenkins, 2002). Con la aparición e incremento de grandes sociedades anónimas, la RSE tuvo un significado más amplio que filantropía y caridad. La definición adoptada en este artículo es que la RSE significa: “[...] las prácticas de la corporación que, como parte de la estrategia corporativa, en complementariedad y apoyo de las más importantes actividades empresariales, busca evitar daño y promover el bienestar de "stakeholders" (clientes, proveedores, empleados, fuentes financieras, la comunidad, el gobierno y el medio ambiente) a través de cumplir con reglas, regulaciones y voluntariamente ir más allá de ellas" (Vives, 2004:1). 
Esta definición enfatiza prácticas que son involucradas en las estrategias corporativas de la empresa. Entonces, explícitamente afirma que la RSE va más allá de una filantropía; que se dirige a un aspecto operacional general de las empresas, además, se enfatizan prácticas y no solamente actitudes. De esta manera, se aleja de definiciones, frecuentemente promovidas por la comunidad empresarial, que sólo están enfocadas a motivaciones y compromisos. $\stackrel{1}{-}$ Adicionalmente, afirma explícitamente que la RSE no es una alternativa a las reglas y regulaciones, cumplir con ellas, entonces es parte de la RSE, pero ésta va aún más allá.

La definición también se aleja de aquéllas que tienen que ver con el enfoque que privilegia al voluntariado, por ejemplo la definición sugerida por la Comisión Europea. $\underline{2}$ Desde mi punto de vista, la definición propuesta por Vives (2004) es más compatible con una perspectiva de la RSE con una visión que no solamente incluye la empresa individual sino el desarrollo en general.

\section{3. ¿Negocio, ética o economía-política? Acercamientos a la RSE}

Antes de presentar mis argumentos e introducir una tercera perspectiva voy a hacer un acercamiento a las dos maneras más comunes de abordar el asunto de la RSE: La ética empresarial y la gesti ón empresarial.

\section{1 . RSE como un buen negocio}

En los acercamientos a la RSE basados en la perspectiva de gestión de negocios, existen tanto estudios analíticos como normativos. El artículo de Brammer y Millington (2003) sobre la inclusión de la comunidad en actividades empresariales y su relación de la forma de organización de la empresa, es un buen ejemplo de estudios analíticos. Ellos encuentran que la forma de organización tiene un impacto en la inclinación de envolver a la comunidad en las actividades empresariales.

Acercamientos normativos tienen el propósito de afirmar el "caso empresarial para la RSE". El argumento mas sólido es que introducir medidas para mejorar la gestión social y ambiental de la empresa no solamente es éticamente correcto, sino que también es un buen negocio.

Este argumento se encuentra también entre inversionistas responsables (IRS) y empresas que se involucran en comercio ético. Los IRS invierten en empresas que toman 
su responsabilidad social, y afirma que las empresas que tienen un buen desempeño de RSE también son las más rentables (Klausen, 2000). Frecuentemente se encuentran argumentos como lo siguiente: “Estas empresas [con altos estándares de RSE] frecuentemente son muy rentables, exactamente porque están administradas por personas modernas, dedicadas, que ven las posibilidades de combinar rentabilidad económica con la protección del medio ambiente" (Storebrand Kaptialforvaltning, 2003).

Empresas que usan muchos recursos para desarrollar informes sobre "la triple línea base" a pesar de que pocas personas realmente leen los informes, utilizan esos mismos argumentos. El concepto "la triple línea base" describe tres tipos de consecuencias de las actividades de las empresas que se deben incluir en la cuenta o informe anual: superávit económico, impacto ambiental e impacto social. 3 Ahora, se discute cada vez más lo que se llama "una crisis de hacer informes" y "una fatiga de intereses". La CSR Europa (2002) señala que las empresas sienten que muy poca gente lee los informes sobre el desempeño de la RSE. A pesar de eso, las empresas afirman que producir informes vale la pena porque el proceso interno de elaborar estos informes aumentan la moral y conciencia en la empresa, lo cual beneficia no sólo el trabajo con RSE, sino la operación de la empresa en general.

Hasta hoy no hay consenso sobre el tema; que si empresas con altos estándares sociales y ambientales también son más rentables que otras. Las experiencias son mixtas, pero todavía hay muchos errores en los instrumentos de comparación (Klausen, 2000). Por ejemplo: Entre los años 2000 y 2002 los fondos éticos crecieron menos (o perdieron más) que carteras comparables. Empero, esto se puede explicar por su sobre-exposición en empresas de tecnología de informática. Muchos fondos éticos sufrieron cuando la "burbuja de las industrias de tecnología de informática" se rompió. Sin embargo, también el fondo ético más conocido - el Dow Jones Sustainability Index (DJSI) -, que se basa en el principio de ser "los mejores de la clase" y por eso no deberían ser vulnerables a cambios entre sectores, ha perdido más, durante la reciente recesión, que el Dow Jones General Index (DJSI, 2003). Por otro lado, otros estudios muestran que las "carteras éticas" crecen más que el mercado general en largo plazo (J oly, 1999; Holme y Watts, 2002).

Cuando uno pone las experiencias en la balanza, se ve que hay un poco más de evidencias sobre, qué empresas con altos estándares de RSE también son más rentables. Sin embargo, la mayoría de los analistas están de acuerdo que se necesitan también argumentos éticos para la RSE. ¿Cuáles son estos argumentos?

\subsection{RSE como buena ética empresarial}


No hay consenso al leer lo que se escribe sobre la ética empresarial puesto que la RSE como está formulada en el debate actual es éticamente razonable. Milton Friedman sostiene que la responsabilidad moral de la empresa se limita a "usar sus recursos con el fin de dedicarse a actividades que incrementan su superávit" (Friedman, 1962), y este argumento todavía es considerado válido por muchas actores. La revista The Economist (2002) sostiene, por ejemplo, que la RSE es un concepto peligroso, 4 mientras que la filósofa Elaine Sternberg señala que las empresas deben evitar "malgastar recursos por objetivos que se llaman "responsabilidades sociales" [que] por su naturaleza son malas para los negocios" (Sternberg, 2001:12).

Según este punto de vista, las empresas tienen responsabilidad hacia sus inversionistas, y sólo cuando produzcan rentablemente estarán cumpliendo con su responsabilidad social. Esto es un concepto estrictamente utilitario, además, se basa en una perspectiva liberal de la sociedad: cuando cada actor desempeña su rol precisamente definido, le sirve también a la sociedad en general. El papel de las empresas es producir bienes y servicios para producir un superávit, y sus obligaciones sociales no se extienden más allá de lo que está establecido en las leyes del país.

Este punto de vista está también apoyado por los que están en contra del uso de la "presunción de constancia" (que si en un principio se aplica a una área de la vida social, tiene que aplicarse también a otras) en la ética empresarial. Una economía de mercado está fundada en la idea que las personas se relacionan como vendedores y compradores. Aquí se consideran las otras personas solamente como medios para un fin. Si eso no fuera el caso, la idea de mercado no funcionaría. Aplicando por ejemplo el principio de Kant que nunca se deben tratar a las otras personas como medios por fines - el mercado no funcionará. Entonces, códigos éticos adecuados son sensitivos al área de la vida social (Phillips, 1993).

Muchos filósofos rechazan el punto de vista de Friedman. Una razón es que las corporaciones actualmente desempañan un papel mucho más amplio en nuestras vidas que en el tiempo de Friedman. En la introducción de un tomo sobre ética empresarial de Alan Malachowski (2001:1) dice: “Las empresas no solamente son las proveedores principales de recursos materiales para la vida fuera de las puertas de la compañía". La esfera empresarial se ha ampliado y ésta incluye todos los aspectos de la existencia humana. Una gran parte de nuestro ambiente natural esta convirtiéndose en una construcción empresarial. Y nosotros mismos parecemos estar destinados a ser criaturas formadas por negocios, programadas en nuestros escondrijos mas profundos del mercado 
y de agendas comerciales".

Otros filósofos, basándose en Aristóteles, afirman que las empresas deben ser consideradas como una especie de comunidades sociales, y no como elementos independientes en la máquina social que funciona según un misterio mágico del mercado. Los "Aristotelianos" consideran que las empresas son instituciones humanas criadas para servir al ser humano. Entonces, tenemos que exigir más de las empresas de que solo funcionan como una manera de hacer dinero. La Ética Empresarial tiene que ver con promover virtudes en la búsqueda de la felicidad máxima (Solomón, 1993). En consecuencia se rechaza el "minimalismo" de Friedman por dos razones. Primero, porque no logra ver las empresas como instituciones humanas, con una responsabilidad de proveer empleos, y que de oportunidad a las personas de vivir una vida integra y virtuosa. Segundo, no logra ver las empresas como partes integradas de la sociedad con una responsabilidad de proveer enriquecimiento para la comunidad en general.

Sin embargo, aunque los Aristotelianos manifiestan que las empresas tienen una responsabilidad más amplia que solo producir un superávit, no nos proporcionan claros principios al respecto. ¿Qué significa esta responsabilidad? hay que preguntarse y además, ¿que es la felicidad máxima? Hay que tomar en cuenta que lo que la empresa considera la búsqueda de la felicidad máxima, otras personas pueden considerarlo como una violación de su forma de vivir. $\underline{4}$

Los Habermasianos intentan superar este problema con aplicación de la ética de discurso. Para ellos la meta final de las actividades es menos importante que el proceso. La meta es crear una situación donde las distintas opiniones puedan ser formuladas y consideradas. Por medio de este tipo de reflexión ético, se puede desarrollar un consenso sobre el comportamiento adecuado. Para aplicar la ética discursiva de Habermas en la empresa hay que asegurar un diálogo abierto dentro la empresa. Pero también hay que promover el diálogo con interesados secundarios (stakeholders).

Este tipo de ética tiene actualmente mucha influencia en círculos empresariales y resulta de un foro de "diálogo de interesados" como una manera de mejorar el desempeño de la RSE en la empresa. Sin embargo, hay problemas con esta perspectiva también. Primero, hay dudas de que sea factible una situación ideal de discurso donde las opiniones pueden ser intercambiadas sin obstáculos. Siempre hay obstáculos en términos de igualdades de poder entre los participantes, diferencias de lenguas, existencia de dependencias, entre otros. Segundo, no se demanda mucho de la empresa; tampoco se dice nada sobre responsabilidades $m$ ínimas que deban ser tomadas. 
Una manera alternativa de abordar el problema es buscando principios con los cuales cualquier persona, independientemente de su posición en la sociedad, puede aceptar. En otras palabras, se buscan principios que produzcan un adecuado equilibrio entre lo favorable y en contra de las personas afectadas. Este es el método de John Rawls en su intención por un tipo de ética deontológico, con lo cual el mensaje más importante es que las instituciones de la sociedad deben ser organizadas de una manera tal que asegure los intereses de los más pobres. Cada persona debe tener derechos políticos y civiles, pero cada persona debe también, tener las mismas oportunidades de obtener posiciones en la sociedad. Inequidad económica solamente puede ser aceptada si un incremento en los beneficios para los ricos asegura que la situación para los pobres también mejora (Rawls, 1972 [1992]) Para lograr un acuerdo sobre qué tipo de instituciones preferimos en la sociedad, Rawls propone una lógica contractual y nos pide imaginarnos que no sabemos que posición y lugar vamos a ocupar en la sociedad. Desde este "velo de ignorancia", donde podríamos ocupar el lugar de los más pobres de la sociedad, vamos a elegir nuestras instituciones.

La teoría de Rawls trata específicamente instituciones políticas, no empresas y habla de principios generales (por ejemplo leyes), y no de un cambio en la actitud de los empresarios. Sin embargo, se puede usar este principio para elaborar principios generales para las empresas. Podemos preguntarnos: ¿Vamos a decidir qué responsabilidad tienen las empresas para promover el bienestar humano, sin saber si somos accionistas, empleados, gerentes, consumidores, abastecedores, o miembros de la comunidad, que tipo de responsabilidad social queremos darle a la empresa entonces?

Es muy probable que la respuesta sea dependiente del contexto. La responsabilidad social de la empresa sería más amplia en situaciones donde no hay muchas organizaciones con la voluntad y la capacidad de promover el bienestar de los individuos. Donde no existe esta responsabilidad, por ejemplo en un Estado con poca capacidad de proveer el bienestar de su pueblo, la empresa tiene más responsabilidad por el bienestar de sus empleados, que en un país que ofrece beneficios generosos y bienestar social.

En suma, este tipo de argumento ético requiere que las empresas tomen una responsabilidad más allá de lo que la ley les obliga, y también más allá de los impactos directos de la empresa. Esto no solamente demanda que las empresas se comporten responsablemente, sino que también presionan al gobierno para que actúe más responsable. Incrementando la responsabilidad de la industria de un "nivel de micro" a un "nivel de macro". Llevaría en otras palabras, una asignación a las empresas, a un rol 
estrictamente político (Haufler, 2001).

El argumento de Rawls también se podría usar como una justificación para la introducción de la economía política en la discusión de la RSE en la ética empresarial. Podríamos en otras palabras preguntarnos: ¿Qué tipo de instituciones y qué tipo de organizaciones sociales elegiremos, si queremos asegurar que las empresas tomen su responsabilidad, y que elegiremos sin conocer nuestra posición en la sociedad? Para dar una respuesta a esta interrogante, tenemos que saber más sobre como las diferentes instituciones y formas de organización de un estado, que afectan el comportamiento de las empresas.

\subsection{RSE como economía-política}

La disciplina académica que ha tratado más profundamente los asuntos mencionados anteriormente, es la economía-política. Hay varias "escuelas" de economía-política, pero una característica de todas es que en el análisis del desarrollo económico no incluye solamente variables económicas, sino también actores políticos con sus intereses, e instituciones que son mediadoras de los intereses políticos. Sin embargo, las diferentes maneras de analizar éstas, son distintas y tendrá que ver con la percepción de la constitución de intereses de los actores, la naturaleza de las instituciones y el nivel de análisis. En la literatura académica de los Estados Unidos, la perspectiva de "actores racionales" ven a los actores como buscadores racionales de utilidad, y a las instituciones como respuestas racionales a problemas de coordinación, predominando la disciplina de la economía política por mucho tiempo. Planteando en este artículo otra perspectiva de economía-política elaborada por Gamble et al. (1996), quienes ven a los actores no (sólo) como buscadores racionales de utilidad, sino tambi én como actores afectados por normas, ideas e instituciones. Además, ven las instituciones no solamente como árbitros neutrales y respuestas a problemas de coordinación, sino también como instrumentos de poder. Al mismo tiempo, analizan el desarrollo económico y político dentro un marco de la economía global, y no solamente la economía de un solo Estado.

Hay grandes variaciones dentro de esta perspectiva, a continuación se elaboran dos hipótesis sobre la RSE basadas en dos ramas de ella:

\section{* RSE, sectores y segmentos de industrias}

La primera hipótesis es que la probabilidad que una empresa introduzca buenos estándares ambientales, estándares laborales, cumpla con reglas y regulaciones y trabaje 
por mejora del bienestar de la comunidad, depende del sector y segmento en el cual la empresa opera. Mientras que la perspectiva económica dominante está solamente preocupada por los aspectos cuantitativos del crecimiento económico, de las políticas económicas, y también de aspectos cualitativos del ser humano (personalidad, ética, habilidades), y por eso también aspectos cualitativos del crecimiento (Hveem y Reinert, 1998).

Una línea económica basada en Schumpeter (1934) argumenta que para comprender el fortalecimiento del crecimiento económico, tenemos que entender no sólo como lograr precios correctos o un buen clima para inversión favorable; innovación y conocimientos son los mayores impulsos para crecimiento económico. La economía no es estática, como se le considera en la economía neo-clásica, sino un sistema dinámico en el cual nuevas tecnologías y nuevos productos reemplazan continuamente a los antiguos. Para que la industria adopte nuevas tecnologías, hay que incrementar el volumen de producción. Bajo condiciones de libre comercio, resulta en una expansión de bienes con un alto contenido de tecnología. Las economías que se enfoquen en actividades con bajos niveles de tecnología experimentan una reducción constante de rentas y niveles de sueldos. En otras palabras, los que se especializan en producir bienes con poca innovación tecnológica, participan sobre todo en una competencia por precio, y están en el largo plazo “especializándose en ser pobres” (Hveem y Reinert, 1998).

La competencia real de costos es factible solamente en una economía globalizada. La búsqueda de mano de obra barata no tiene límites. En 1980 afirmaron Fröbel et al. que una nueva división de trabajo internacional ya era evidente. Esta involucra la concentración de capital, innovación tecnológica, producción y gestión de alta tecnología en el centro (las partes ricas del mundo ubicados en el Norte del mundo) y la fase intensiva de mano de obra en la periferia (Fröbel et al, 1980). Esta imagen se basa en una diferencia entre un centro geográfico del mundo constituido por los países ricos y desarrollados, y una periferia constituida por países pobres y subdesarrollados. Hoy, la teoría es menos relevante porque los capitales transnacionales combinan "estrategias de mudanza" al Sur y el uso de mano de obras inmigrante en el centro (en el Norte). Al mismo tiempo, segmentos monopolizados y de alto valor de las industrias se muda al Sur (Robinson, 2003). En otras palabras, hay una diferenciación dentro de las sociedades en el Norte y el Sur, entre segmentos de industrias que son de alta tecnología y alto valor agregado y segmentos con baja tecnología y bajo nivel de sueldos.

A pesar de que la perspectiva arriba mencionada habla de niveles de salarios y esto no tiene mucho que decir sobre la calidad del empleo, basada en esta teoría se puede 
formular la hipótesis de que: segmentos de industrias con bajo nivel de tecnología también son partidarias de peores políticas de RSE. Esto implica que para estrategias políticas que no es suficiente intentar de motivar Ideres empresariales para introducir políticas de RSE. También, se deben aplicar estrategias para evitar que un país o área sea atrapado en los segmentos más bajos de las cadenas de producción, con poca tecnología y donde las empresas compiten por producir más barato.

Sin embargo, no es muy probable que los pobres en el mundo tengan la posibilidad de participar en segmentos, con alta-tecnología de industrias, en el futuro previsible. Por eso, todavía es importante que partes de las industrias que operan en segmentos más bajos también tome responsabilidad social. En lo siguiente voy a discutir como facilitar esto.

\section{* RSE, Estados y empresas}

Un principal enfoque en la literatura de economía-política es la relación entre el estado y el sector privado. Estados, mercados y sociedad no son entidades separadas. El estado esta basado en el sistema económica y social. Es por eso que cualquier cambio en el estado está reflejado en la sociedad y la economía y vise-versa (Underhill, 2003). Un mercado funcionando sin el Estado se ve como una ilusión. Al mismo tiempo, en contradicción a la economía neo-clásica, perspectivas de economía política discuten no solamente la participación del estado en la economía, sino también la naturaleza de los enlaces entre el Estado y el sector privado.

El punto de partida son los cambios en el capitalismo global durante los últimos años. Un aspecto de esto es" la retirada" del Estado en funciones claves de la sociedad. Esto se ha interpretado como el retiro del Estado en general (Strange, 1996). Sin embargo, ahora los mejores estudios empíricos muestran que la globalización no socava el Estado (Castells, 2004; Guillén, 2001). Lo que estamos experimentando es sobre toda una reorganización del estado (Amoore et al., 1997).

Esta reorganización se ha interpretado de maneras contradictorias. Primero, se le ha interpretado como la transnacionalización del Estado (Cox, 1981). Esto significa que los Estados cada vez más ajustan sus prácticas-políticas nacionales a las exigencias de la economía global. La transnacionalización del Estado involucra una reorientación de los servicios del Estado al capital privado, y de fracciones nacionales a las fracciones transnacionales del sector privado (Robinson, 2003:144). En otras palabras, la idea de transnacionalización del Estado tiene que ver con la idea del surgimiento de una clase transnacional involucrada en la producción global y que maneja circuitos globales de 
acumulación de capital (Gill y Law, 1989; Sklair, 2001).

El Estado transnacional se define como: "una constelación particular de clases y relaciones vinculadas a la globalización capitalista y al avance de una clase capitalista transnacional, encarada en instituciones políticas diversas [...] el avance de una clase capitalista transnacional implica la reorganización del Estado en cada nación, e implica al mismo tiempo, el surgimiento de instituciones económicas y políticas verdadera transnacionales" (Robinson, 2003:43). Esta descripción de las condiciones actuales no le deja mucho espacio al Estado para desempeñarse autónomamente. El Estado ha sido y es todavía un instrumento para las clases dominantes, con la diferencia actual que las clases dominantes son de carácter transnacional.

Segundo, se le ha interpretado como el surgimiento de Estados reguladores. Este concepto pretende describir la esencia de las transformaciones de la economía capitalista en los finales del siglo pasado (Jordana y Levi-Faur, 2004). Usando una metáfora marítima, se afirma que el Estado regulador ya no pretende "remar" la economía (por ejemplo a través de empresas públicas), pero intenta "conducirla" (Braithwaite, en Jordana y Levi-Faur, 2004). Regulaciones en este sentido no solamente significa emitir leyes, sino también guiar la economía de maneras menos formales.

La capacidad del Estado para "remar" la economía es la caracter ística principal que se encuentra en la literatura sobre el milagro económico en Asia-Este (Evans, 1995; Weiss, 1998). Una conclusión que se puede leer en la literatura es que el Estado autónomo, con enlaces formales e institucionalizados en la sociedad (sobre todo en el sector privado), lo que Peter Evans (1995) llamó “Estado enraizado" fue clave para entender el éxito y desarrollo de las economías Asiáticas. Este tipo de Estados aseguraba comunicación e intercambio de información que ayudaba a las empresas y Estados a trabajar juntos para el desarrollo. Esta perspectiva nos puede ayudar también a entender la capacidad de diferentes Estados para "conducir" la economía. Se puede formular la hipótesis que un Estado caracterizado por una "autonomía enraizada" también tiene mejor posibilidades de motivar a las empresas para que sean actores más responsables.

El concepto de un Estado regulador implica que el Estado es más autónomo que el Estado transnacional. Desde mi punto de vista, ni el uno ni el otro describen mejor la realidad. Lo cierto es que se debe facilitar el surgimiento de Estados reguladores con capacidad no sólo para regularizar y facilitar la economía capitalista, sino también para proteger los derechos humanos y el medio ambiente, será una tarea clave si queremos facilitar el surgimiento de empresas responsables. 
En conclusión, aplicar la RSE desde esta perspectiva significa no solamente que debemos promulgar en las empresas existentes cambios en su conducta, sino también se debe pensar en cuáles tipos de industrias y segmentos son más atractivos para el bienestar de los seres humanos y el medio ambiente, y qué tipo de regulaciones - nacionales, regionales y globales - son necesarios para mejorar la situación de ellos.

Al final, para entender los impulsos en la introducción de políticas de RSE, se requiere de una combinación de perspectivas micros y macros, lo cual representa la gestión de las empresas, ética empresarial y políticas económicas respectivamente. De la perspectiva de gestión de empresas y ética empresarial podemos aprender algo sobre el comportamiento de una empresa dentro un marco fijo. De la perspectiva de políticas económicas podemos aprender algo sobre como desarrollar el marco.

\section{RSE y el modelo de desarrollo actual en América Latina}

En esta sección revisaremos algunos de los datos existentes sobre RSE para investigar las hipótesis arriba mencionadas. La primera pregunta es si las empresas con industrias y segmentos de baja tecnología violan principios de buena conducta de RSE más frecuentemente que otras. Es complicado responder a esta pregunta. Primero, algunos de los índices más importantes sobre comportamiento de RSE son divididos en sectores. EI Dow Jones Sustainability Index, por ejemplo, solamente presenta las mejores empresas en cada sector, y no compara sectores. Segundo, muchas empresas están involucradas en actividades en varios sectores, algunas son de alta tecnología y otros de baja tecnología. Tercero, la mayoría de los fondos éticos, no publican sus listas de empresas excluidas, lo cual significa que no vamos a saber quienes son los peores actores.

Hay algunos datos que nos pueden servir. Algunos inversionistas publican listas de empresas excluidas por violaciones de normas de buena conducta. Un ejemplo es el Fondo de Previsión Noruego, KLP, que sujeta todas sus inversiones a criterios éticos. KLP publica los nombres de las empresas excluidas así como las razones por las cuales fueron excluidas. Su mecanismo de filtración se basa en acuerdos y convenciones internacionales en las áreas de derechos humanos (inclusive derechos de los niños), derechos laborales, medio ambiente, venta ilegal de armas y corrupción. $\underline{5}$ Las empresas pueden ser excluidas si se encuentran evidencias aprobadas por organizaciones de las Naciones Unidas sobre la violación de acuerdos y convenios. Aunque KLP es una inversionista pequeña, varios fondos más grandes utilizan el mismo método sin publicar la lista de las empresas. Por eso es probable que las empresas listadas por KLP sean representativas de empresas con 
mala conducta de RSE.

En la lista de la KLP de empresas excluidas se puede observar algunas tendencias. Primero, la mayoría de las empresas excluidas están en esta lista por violaciones de acuerdos y convenios ocurridas en América Latina. En la lista editada en 2003, la mitad de las violaciones habían ocurrida en México, Centroamérica y Colombia. Ninguna de las empresas fueron excluida por violaciones ocurridas en los Estados Unidos o Europa. Entre las empresas excluidas en 2004, 61\% fueron excluidas por violaciones ocurridas en América Latina. (Ecuador, Colombia, Costa Rica, México y Guatemala). Segundo, dos tipos de actividades dominan las lista de empresas excluidas: industrias extractivas (sobre todo petróleo y minería), y "ensambladoras" incluyendo "maquiladoras" e industria de micro tecnología. Aunque también se han excluido empresas de alta tecnología, por ejemplo Siemens, generalmente son excluidas por violaciones en sus plantas de producción que no demandan altas calificaciones $\underline{6}$.

\section{RSE y la relación estado-empresarial en América Latina en una perspectiva comparativa}

La segunda pregunta es: ¿En que Estados tienen las empresas un mejor compartimiento de RSE? Dar una respuesta a esta pregunta se dificulta por obstáculos de índole metodológico. Primero, es bien conocido que hay muchos países que son violadores de los derechos humanos y de convenciones de las Naciones Unidas. Por ejemplo, el Dow Jones Sustainability Index excluye empresas con inversiones significantes en una serie de países. Se debe mencionar que el origen del surgimiento de la idea actual de RSE se dio parcialmente debido al boicot internacional de Sudáfrica durante el sistema de apartheid. La idea de internacionalización de inversiones a países con sistemas institucionales débiles es parte del motivo para promover la RSE.

Segundo, el fuerte interés por las RSE está relacionado con la disminuida relevancia de fronteras nacionales. Por ejemplo, muchas veces se afirma que el impulso de la RSE es por la globalización de los medios de comunicación y las ONGs (Jenkins, 2002). Sin embargo, perspectivas de políticas económicas nos debe hacer presente que el estado nacional todavía es relevante.

Tercero, tenemos que clarificar si hablamos en la relación Estado-empresa, en el Estado anfitrión o en el Estado de origen de la empresa. Enfocándome en la última relación, he estudiado las actitudes de los líderes empresariales noruegos y centroamericanos 7 . 
Los líderes empresariales noruegos operan en un contexto muy regulado. Hay, por ejemplo, regulaciones estrictas sobre prácticas ambientales y laborales. El Estado y las grandes empresas siempre han tenido una relación muy íntima, pero el Estado tradicionalmente ha tenido más poder. Aunque las empresas noruegas tienen altos estándares en lo ambiental y laboral, y existe una idea común en éstas sobre su comportamiento internacional en lo referente a estos aspectos son mejores que en las empresas de otros países, pero según la conclusión de un estudio de sus estrategias internacionales de RSE las empresas noruegas tienen un comportamiento deficiente. En comparación con empresas de otros países europeos, las empresas noruegas están retrasadas en cuanto a sus informes sobre su conducta social, $\underline{8}$ no usan mucho los estándares internacionales, y no forman alianzas con las ONGs con mayor frecuencia 9 .

Líderes empresariales en Noruega tienen diferentes opiniones con respecto a la relación entre el rol del Estado en Noruega y el comportamiento de empresas en el extranjero. Los líderes opinaron por un lado que las empresas noruegas están acostumbradas a ser reguladas y tienen una relación muy íntima con el movimiento laboral. Por eso tienen una ventaja significante con respecto a trabajo en el campo de la CSR. En otras palabras, están acostumbrados a tomar otros factores en cuenta además, del superávit en sus operaciones diarias. Por la tradición de regulación, las empresas noruegas siempre cuentan que vendrán nuevas regulaciones y tratan de implementarlas antes de que sean introducidos por ley y así tener una ventaja en relación con sus competidores. Asimismo, el fuerte compromiso internacional del Estado Noruego por la paz y desarrollo en general se transmite a las empresas, según dicen algunas de ellas. También, el fuerte rol del Estado es considerado como un importante factor detrás del nivel relativamente bajo de corrupción en Noruega.

Por otro lado, se considera que gracias al sistema noruego de economía mixta, con regulaciones estrictas con respecto a derechos laborales y protección al medio ambiente, y una relación semi-corporatista entre la mano de obra, capital y Estado, la sociedad Noruega ha sido protegida de unas de los efectos más duras del capitalismo global. Como consecuencia de esto, las empresas noruegas han sido menos expuestas a críticas que otras empresas transnacionales han sido en relación a las operaciones de la economía global.

La literatura histórica de las empresas ya empresarial en América Latina muestra que en los distintos países latinoamericanos hay diferentes relaciones entre el Estado y las empresas, y también diferentes actitudes de los Íderes empresariales (Miller, 1999). En algunos países hay Estados relativamente fuertes, y en otros ha sido más que todo un 
instrumento de las élites empresariales. También la ética empresarial se desarrolla diferentemente en los distintos países. Sin embargo, hay algunos elementos en común. Coutinho de Arruda de la Escuela de Negocios de Sao Paolo afirma que en América Latina, la ética de las empresas incluye integridad y responsabilidad social, simbolizando el compromiso de las empresas para la sociedad. Sin embargo, la falta de institucionalización y la falta de sumisión de leyes que regulan la corrupción, los derechos laborales y la protección del medio ambiente, resulta en el actitud general descrito por Arruda la manera siguiente: "Un dicho muestra la cultura no-ética dominando en muchas empresas de los países hispanohablantes: El que no tranza no avanza" (Arruda, 1997: 1598).

Arruda (1997:1599) afirma también que las empresas de América Latina, en contradicción con las noruegas, frecuentemente consideran empresas extranjeras más éticas que las locales: "En muchos aspectos, son los extranjeros que muestran que principios éticos son las reglas del juego que el empresario debe respectar. Por ejemplo, algunas empresas mexicanas buscan contrapartes que puedan aportar no tan solo tecnología, capital y oportunidades para entrar en nuevos mercados, sino también estándares éticos para formar empresas conjuntas".

Investigaciones sobre la RSE en América Latina nos dan respuestas contradictorias en cuanto a la situación de empresas Latinas en su agenda de RSE. Peinado-Vara (2004) estudia la reciente literatura de la RSE en América Latina y afirma que las conclusiones no son muy consistentes. Enfocando la adopción de estándares (incluyendo SA8000, AA 1000, y GRI) un estudio concluye que América Latina está retrasada (IADB, 2004). Otro muestra que las economías mas avanzadas (Argentina, Chile y México) están poniéndose al día, pero que las empresas latinoamericanas son muy influidas por ONG's y comunidades empresariales internacionales en su acercamientos a la RSE. Otros estudios muestran que América Latina no está muy atrasada comparada con empresas europeas o estadounidenses (IFC, 2002). Sin embargo, estudios de México y América Central encuentran que la RSE está asociada con filantropía y que sobre todo en las economías más pequeñas y más vulnerables, la norma aún es que a las empresas les interesa tener mano de obra barata y un marco legal "blando" en el área del medio ambiente (Barkin, 2003).

En la mayoría de los estudios sobre RSE prevalecen aquellos que están enfocados en el comportamiento de las empresas internacionales en América Latina, y no en las empresas latinoamericanas. Mis propias investigaciones, entre la elite empresarial de Centro América, muestra más diferencias que similitudes entre las elites de los diferentes países. En el país con el Estado más fuerte y con las mejores relaciones entre empleados, 
empleadores y el Estado - Costa Rica - los Ideres empresariales muestran actitudes más sociales. En Guatemala y El Salvador - en donde el estado tradicionalmente ha jugado un rol mínimo en cuanto a responsabilidad social - las ideas liberales de Milton Friedman y Ludwig von Mises son más influyentes. Desde esta perspectiva las empresas han cumplido con su responsabilidad cuando crean puestos de trabajo y superávit económico (Bull, 2001). Un líder empresarial influyente describió la situación como sigue: Se respetan los derechos de otras personas y se cumple con los contratos. Se cumple con la ley general, pero después se puede hacer lo que quiera. No puedes hacer algo mal, hay que cumplir con estas circunstancias. 10 Por otro lado, se argumentó que las empresas en realidad toman una responsabilidad mayor a la impuesta por la ley, y en general los líderes empresariales consideran que ellos mismos tienen estándares sociales y éticos más elevados que los del Estado.

Líderes empresariales en Costa Rica revelaron un grado más alto de escepticismo sobre la posibilidad de que las empresas ahora toman el liderazgo en el campo social. Se consideraron que empresarios locales todavía tenían que sacarse el "parásito capitalista" que fue tan prevalente durante los años 1960 y 1970. Al mismo tiempo, todos los empresarios entrevistados, incluso aquellos que se encontraron a la extrema derecha del marco político, añadieron que el Estado debe jugar un papel social mas grande que lo que piensan sus colegas en los otros países centroamericanos.

Los hondureños por otro lado, mostraron un cierto conformismo en cuanto a la ética empresarial y a la responsabilidad social de las empresas. Mientras que la mayoría afirmaba que las empresas si tenían una responsabilidad social, muchos estaban desilusionados con respecto a la posibilidad de un comportamiento ético en el marco que ofrecen las instituciones políticas y legales de Honduras.

Sin embargo, lo que más llama la atención es que las opiniones de los líderes empresariales sobre su responsabilidad social son muy similares a sus opiniones sobre cual es la responsabilidad social de los Estados. Mientras que los guatemaltecos y los salvadoreños favorecieron un Estado mínimo y también que la responsabilidad social de la empresa fuera mínima, los costarricenses y hondureños tuvieron actitudes diferentes. Eso revela que hay una conexión entre el rol tradicional del estado y las actitudes de las empresas.

La relación Estado-empresa solamente es un factor entre muchos otros que son importantes si queremos explicar porqué algunas empresas toman más responsabilidad que otras. Sin embargo, según nuestras conclusiones preliminares, la relación Estado- 
empresa, es importante si queremos mejorar el comportamiento social empresarial.

\section{Conclusión}

El propósito principal de este artículo ha sido incluir una perspectiva de económicapolítica en el análisis de RSE. Esta perspectiva no es nueva. Desde este análisis, se puede afirmar que lo que es nuevo en el debate es el enfoque de las empresas como actores y su responsabilidad social voluntaria. Sin embargo, mucha de la literatura actual que ha surgido del "boom" reciente de RSE, nota algunas diferencias sistemáticas entre empresas que se encuentran operando en distintos contextos estructurales. Estos resultados hasta aquí producidos ameritan que se sigan profundizando en el análisis y discutidos en debate sobre la RSE, que traten sobre todo de aspectos que den cuenta de cómo organizar nuestra sociedad - e incluyendo las empresas - para que podemos aumentar el bienestar para común.

Actualmente, se discuta la RSE en relación de tratados de libre comercio, por ejemplo el que firmaron los países en Centro América y los Estados Unidos (CAFTA). Esto es controversial porque tiene el potencial de ser usado como una medida proteccionista. Según la perspectiva de la economía-política, no se debe enfocar solamente en medidas de RSE en las FTAs, sino también en qué tipo de sectores son favorecidos por las FTAs, y que tipo de relación entre el Estado y las empresas resultará. A fin de cuentas, eso puede ser mas importante para la contribución de las empresas en el desarrollo general.

\section{Notas}

1. Un ejemplo es la definición, promovido por el Consejo Mundial Empresarial sobre Desarrollo Sostenible (WBCSD) (sigla en ingles) es: "El compromiso de la empresa de contribuir al desarrollo económico sostenible, trabajando con los empleados, sus familias, la comunidad local y la sociedad en general para mejorar su calidad de vida" (Holliday et al., 2002: 103).

2. Esta es así: "La RSE es un concepto en el cual las empresas integran consideraciones sociales y ambientales en sus operaciones empresariales y en sus relaciones con sus "stakeholders" de una manera voluntaria" (European Comisión, 2002: 7).

3. Impacto social tiene que ver con la preocupación de las empresas por sus empleados así como la sociedad en general. Internamente, con mejorar las condiciones de salud, el medio ambiente y seguridad, darle a los empleados oportunidades para educación, y evitar discriminación de cualquier grupo de empleados (por ejemplo minorías étnicas, minorías de género). Externamente, puede involucrar una serie de temas como políticas de derechos humanos, 
condiciones laborales en empresas abastecedoras, corrupción, patrocinio y contribuciones a la comunidad local.

4. Vease The Economist, Diciembre 12-24 (2002): Ethical reporting irresponsible: The dangers of corporate social responsibility. The Economist y otros autores frecuentemente atribuyen este punto de vista a Adam Smith, indicando lo siguiente: “No esperamos tener nuestra cena por el benévolo del carnicero, del cervecero y del panadero, sino por su preocupación por sus propio intereses. Nos dirigimos a ellos, no a su humanidad, sino a su preocupación por ellos mismos" (de "An Inquiry into the Nature and Causes of the Wealth of Nations, 1776; republicado en London: Dent 1919, Vol. 1, p. 13). Sin embargo, como afirma Sen (1993), esta citación esta sacada de su contexto y no ofrece una adecuada representación sobre lo que Smith intentaba señalar.

4. Piensa por ejemplo en una empresa que construye una carretera cruzando un pueblo. Aunque la empresa puede ver esta carretera como necesaria para facilitar el desarrollo del pueblo, otras personas pueden verla como una intrusión y como algo que excluye usos tradicionales de la tierra. Por una buena defensa de la perspectiva Aristoteliana de la ética, ver Nussbaum (2000).

5. Esta se funda en la Declaración Mundial sobre los Derechos Humanos, La Convención sobre Derechos de los Niños, varias convenciones de la Organización Mundial de Trabajo (OMT), la Convención de Rió sobre el desarrollo y el medio ambiente, la Convención sobre diversidad biológica, la Convención de Minas antipersonales, y la Convención Contra Sobornos y Corrupción.

6. Se debe notar que Siemens fue una de las pocas empresas que empezó a investigar la razón por su exclusión con la visión de mejorar la situación (Entrevista, Analista, KLP, 6.3.2003).

7. El estudio en Noruega fue realizado sobre la base de entrevistas a 10 líderes empresariales, así como el análisis de documentos oficiales y literatura secundaria. El estudio en Centroamérica fue hecho como parte de las investigaciones para un libro sobre las políticas de reforma de las telecomunicaciones en América Central. En total, se realizan aproximadamente 25 entrevistas a líderes empresariales de Honduras, Costas Rica, Guatemala y El Salvador.

8. Producir informes anuales sobre su conducta ambiental es obligación por ley en Noruega.

9. Ver Bull (2003) para más detalles.

10. Entrevista, Ciudad de Guatemala 23.10.00.

\section{Referencias Bibliográficas}

1. Amoor, Louise, Richard Dodgson, Barry K. Gills, Paul Langley, Don Marshall y Iain Watson (1998), Overturning "Globalisation": Resisting the teleological, Reclaiming the 
“Political”. New Political Economy. Vol. 2. No. 1, pp. 179- 195.

2. Arruda, Maria Cecilia Coutinho (1997), "Business Ethics in Latin America". J ournal of Business Ethics, 16: 1597-1603.

3. Barkin, David (2003), Corporate Social Reposnsibility in Mexico: The Tyranny of a Concept?" Summaries of Presentations made at the UNRISD Conference on "Corporate Social Responsibility and Development: Towards an New Agenda?", 17-18 November 2003, Geneva.

4. Bowen, Howard A. (1953), Social Responsibility of the Businessman. New York: Harpen and Brothers.

5. Brammer, Stephen y Andrew Millington (2003), The Effect of Stakeholder Preferences, organizational Structure and Industry Type on Corporate Community Involvement, Journal of Business Ethics (45) 2003, pp. 213-226.

6. Bull, Benedicte (2001), From Ludwig von Mises to Financial Mitch: The transfer and transformation of neo-liberalism in Guatemala, Paper presented at the Third International CISS/ISA Millenium Series Conference, Heidelberg 25-26 June, 2001.

7. Bull, Benedicte (2003), Aid, Power and Privatization: The Politics of Telecommunication Reform in Central America, Edward Elgar Publishing, forthcoming.

8. Castels, Franscis (2004), The Future of he Welfare State - Crisis Myths and Crisis Realities, Oxford: Oxford Unviersity Press.

9. Cox, R.W. (1981), Social forces, states, and world orders: beyond international relations theory. Millenium: Journal of International Studies, 10, 2, 126-155.

10. CSR Europe (2002), I mpacts of Reporting - CSR Europe. Available at: http:// www.csreurope.org

11. Davis, Keith (1963), Can Business Afford to Ignore Social Responsibilities? California Managemetn Review, Spring (2), pp. 70-76.

12. DJ SI (2003), Dow Jones Sustainability Indexes DJ SI Newsletter 1/2003. 
13. European Commission (2002), Corporate social responsibility: A business contribution to sustainable development. European Commission, Directorate for Employment and Social Affairs, Unit. D.1., July 2002.

14. Evans, Peter (1995), Embedded autonomy: states and industrial transformation. Princeton: Princeton University Press.

15. Friedman, Milton (1962), Capitalism and Freedom. Chicago: University of Chicago Press.

16. Fröbel, F., Je. Heinrichs y O. Kraye (1980), The New International Division of Labour, Cambridge: Cambridge Unviersity Press.

17. Gamble, Andrew, Anthony Payne, Ankie Hoogvelt, Michel Ditrich y Michael Kenny (1996) Editorial: New Political Economy, New Political Economy, Vol. 1, No.1, pp. 5- 11.

18. Gill, Stephen y David Law (1989), "Global Hegemony and the Structural Power of Capital". I nternational Studies Quarterly. 33, pp. 475-499.

19. Gullién, Mauro (2001) Is globalization Civilizing, Destructive or Feeble? A Critique of Five Key Debates in the Social Science Literature, Annual Review of Sociology, 27, 235-260.

20. Haslam, P.A. (2004), The Corporate Social Responsibility System in Latin America and the Caribbean. Policy Paper. Canadian Foundation for the Americas. http://www.focal.ca.

21. Haufler, Virginia (2001), A public role for the private sector. Industry SelfRegulationin a Global Economy. Washington, D.C.: Carnegie Endowment for International Peace.

22. Holliday, Charles O. Jr, Stephan Schmidheiny y Phillip Watts (2002), Walking the Talk. The Business Case for Sustainable Development. Sheffield: Greenleaf Publishing, Itd.

23. Holme, Richard y Phil Watts (2002), Corporate Social Responsibility: Making Good Business Sense. Geneva, Switzerland: World Business Report for Sustainable 
Development.

24. Hveem, Helge y Erik Reinert (1999), Utviklingsideenes utvikling. I Tor-Arve Benjaminsen and Hanne Svarstad (eds.) Samfunnsperspektiver på miljø og utvikling. Oslo: Universitetsforlaget.

25. IADB (2004), Corporate Social Responsibility in Latin America, unpublished report. Washington, D.C.: Inter-American Development Bank.

26. Jenkins, Rhys (2002), Corporate Codes of Conduct: Self-Regulation in a Global Economy, in Unrisd/ NGLS, Voluntary Approaches to Corporate Responsibility: Reading and a Resource Guide, Geneva: UN Non-Governmental Liaison Service (NGLS), pp. 1-60.

27. Joly, Carlos (1999), The Greening of Financial Markets. Paper presented at the Fifth Expert Working Group Meeting on Financial Issues of Agenda 21 United Nations, Nairobi.

28. Jordana, Jacint y David, Levi-Faur (2004), Towards a Latin American Regulatory State? The Diffusion of Autonoous Regulatory Agencies across Countries and Sectors, in Levi-Faur and Eran Vigoda-Gadot (Eds.) I nternational Public Policy and Management: Policy Learning Beyond Regional, Cultural and Political Boundaries, Marcel Dekker, forthcoming.

29. Klausen, Trond Beldo (2000), Etiske Investeringer. Rappport 16:2000, Oslo: Institutt for Samfunnsforskning.

30. Malchowski, Alan (2001), Introduction: Business Ethics Comes of Age, in Alan Malchowski (ed.) Business Ethics: Critical perspectives on business and management. London and New York: Reoutledge, pp.1-4.

31. Melling, Christine og Svenn Are Jenssen (2002), "Fra veldedighet til nødvendighet". En introduksjon til begrepet bedrifters samfunnsansvar. Norges teknisknaturvitneskapelige universitet (NTNU). Institutt for tverrfaglige kulturstudier. Rapport nr. $2 / 2002$.

32. Miller, Rory (1999), Business History in Latin America: an introduction. En Carlos Dávila y Roger Miller (eds.) Business History in Latin America: The Experiences of 
Seven Countries, Liverpool: Liverpool University Press, pp. 1-16.

33. Nussbaum, Martha C. (2000), Women and Human Development: The Capabilities Approach. Cambridge: Cambridge University Press.

34. Peinado-Vara, Estrella (2004), Corporate Social Responsibility in Latin America and the Caribbean, Sustainable Development Department, Technical Papers Series, Washington, D.C.: Inter-American Development Bank.

35. Phillips, Michael (1993), How to think systematically about business ethics. In E.R. Windkler and J.R. Coombs (eds.) Applied Ethics: A reader, Oxford: Basil Blackwell, pp. 185-200 (Har den egentlig fra Machowski).

36. Rawls, John (1972), A Theory of J ustice. Oxford: Oxford University Press (Twelfth Edition 1992).

37. Robinson, William (2003), Transnational Conflicts: Central America, Social Change and Globalization, London and New York: Verso.

38. Schumpeter, Joseph A. (1934), The theory of economic development. Cambridge: Harvard University Press.

39. Sen, Amartya (1993), Does business ethics make economic sense? Business Ethics Quarterly 3 (1), pp. 45-54.

40. Sklair, Leslie (2001), The Transnational Capitalist Class, Malden: Balckwell Publising.

41. Solomon, Robert C. (1993), The aristotelean approach to business ethics: Cooperation and Integrity in Business. Oxford: Oxford University press, pp. 101-111.

42. Sternberg, Elaine (2001), The Importance of Business Ethics. In Alan Malchowski (ed.) Business Ethics: Critical perspectives on business and management. London and New York: Routledge, pp. 10-20

43. Storebrand kapitalforvaltning (2003), Forretningsmessig samfunssansvar. Oslo: Storebrand. 
44. Strange, Susan (1996), The retreat of the state. The diffusion of power in the world economy. Cambridge: Cambridge University Press.

45. Underhill, Geoffrey (2003), States, Markets and Governance for Emerging Market Economies: Private Interests, the Public Good and the Legitimacy of the Development Process, I nternational Affairs, 79 (4), 755-781.

46. Vives, Antonio (2004), The Role of Multilateral Development Institutions in Fostering Corporate Social Responsibility, Sustainable Development Department, Technical Papers Series, Washington, D.C.: Inter-American Development Bank.

47. Vives, Antonio y Estrela Peinado-Vara (Eds.) (2004), Corporate Social Responsibility as a Tool for Competitiveness. Inter.American Conference on CSR Proceedings held in Panama City, Panama 26-28 October 2003, accessed at http: // www.csramericas.org

48. Weiss, Linda (1998), The Myth of the Powerless State. Governing the Economy in a Global Era, Cambridge: Polity Press. 